\title{
ON QUASI-IDEALS AND BI-IDEALS IN TERNARY SEMIRINGS
}

S. KAR

Received 31 May 2005

We introduce the notions of quasi-ideal and bi-ideal in ternary semirings and study some properties of these two ideals. We also characterize regular ternary semiring in terms of these two subsystems of ternary semirings.

\section{Introduction}

Good and Hughes [9] introduced the notion of bi-ideal and Steinfeld [11, 12] introduced the notion of quasi-ideal. Sioson [10] studied some properties of quasi-ideals of ternary semigroups. In [1], Dixit and Dewan studied about the quasi-ideals and bi-ideals of ternary semigroups. Quasi-ideals are generalization of right ideals, lateral ideals, and left ideals whereas bi-ideals are generalization of quasi-ideals.

In [2], we introduced the notion of ternary semiring. Some work on ternary semiring may be found in $[3,4,8,6,7,5]$.

Our main purpose of this note is to introduce the notions of quasi-ideal and bi-ideal in ternary semirings and study regular ternary semiring in terms of these two subsystems of ternary semirings.

\section{Preliminaries}

Definition 2.1. A nonempty set $S$ together with a binary operation, called addition and a ternary multiplication, denoted by juxtaposition, is said to be a ternary semiring if $S$ is an additive commutative semigroup satisfying the following conditions:

(i) $(a b c) d e=a(b c d) e=a b(c d e)$,

(ii) $(a+b) c d=a c d+b c d$,

(iii) $a(b+c) d=a b d+a c d$,

(iv) $a b(c+d)=a b c+a b d$, for all $a, b, c, d, e \in S$.

Definition 2.2. Let $S$ be a ternary semiring. If there exists an element $0 \in S$ such that $0+x=x$ and $0 x y=x 0 y=x y 0=0$ for all $x, y \in S$, then " 0 " is called the zero element or simply the zero of the ternary semiring $S$. In this case we say that $S$ is a ternary semiring with zero. 
Throughout this note, $S$ will always denote a ternary semiring with zero and unless otherwise stated a ternary semiring means a ternary semiring with zero.

Definition 2.3. An additive subsemigroup $T$ of $S$ is called a ternary subsemiring of $S$ if $t_{1} t_{2} t_{3} \in T$, for all $t_{1}, t_{2}, t_{3} \in T$.

Definition 2.4. An additive subsemigroup $I$ of $S$ is called a left (resp., right, lateral) ideal of $S$ if $s_{1} s_{2} i$ (resp., $\left.i s_{1} s_{2}, s_{1} i s_{2}\right) \in I$, for all $s_{1}, s_{2} \in S$ and $i \in I$. If $I$ is both left and right ideal of $S$, then $I$ is called a two-sided ideal of $S$. If $I$ is a left, a right, a lateral ideal of $S$, then $I$ is called an ideal of $S$.

An ideal $I$ of $S$ is called a proper ideal if $I \neq S$.

Proposition 2.5. Let $S$ be a ternary semiring and $a \in S$. Then the principal

(i) left ideal generated by $a$ is given by $\langle a\rangle_{l}=\left\{\sum r_{i} s_{i} a+n a / r_{i}, s_{i} \in S ; n \in Z_{0}^{+}\right\}$,

(ii) right ideal generated by $a$ is given by $\langle a\rangle_{r}=\left\{\sum a r_{i} s_{i}+n a / r_{i}, s_{i} \in S ; n \in Z_{0}^{+}\right\}$,

(iii) lateral ideal generated by $a$ is given by $\langle a\rangle_{m}=\left\{\sum r_{i} a s_{i}+\sum p_{j} q_{j} a r_{j} s_{j}+n a / p_{j}, q_{j}, r_{i}\right.$, $\left.s_{i} \in S ; n \in Z_{0}^{+}\right\}$, where $\sum$ denotes the finite sum and $Z_{0}^{+}$is the set of all nonnegative integers.

Definition 2.6. A ternary semiring (ring) $S$ is said to be zero divisor free (ZDF) if for $a, b, c \in S, a b c=0$ implies that $a=0$ or $b=0$ or $c=0$.

Definition 2.7. A ternary semiring $S$ is called

(i) multiplicatively left cancellative (MLC) if $a b x=a b y$ implies that $x=y$,

(ii) multiplicatively right cancellative (MRC) if $x a b=y a b$ implies that $x=y$,

(iii) multiplicatively laterally cancellative (MLLC) if $a x b=a y b$ implies that $x=y$.

A ternary semiring $S$ is called multiplicatively cancellative (MC) if it is MLC, MRC, and MLLC.

Note 2.8. A multiplicatively cancellative (MC) ternary semiring $S$ is zero divisor free (ZDF).

Definition 2.9 [3]. A ternary semiring $S$ with $|S| \geq 2$ is called a ternary division semiring if for any nonzero element $a$ of $S$, there exists a nonzero element $b$ in $S$ such that $a b x=$ $b a x=x a b=x b a=x$ for all $x \in S$.

Definition 2.10 [2]. An element $a$ in a ternary semiring $S$ is called regular if there exists an element $x$ in $S$ such that $a x a=a$. A ternary semiring is called regular if all of its elements are regular.

\section{Quasi-ideal and bi-ideal in ternary semirings}

Definition 3.1. An additive subsemigroup $Q$ of a ternary semiring $S$ is called a quasi-ideal of $S$ if $Q S S \cap(S Q S+S S Q S S) \cap S S Q \subseteq Q$.

Note 3.2. Every quasi-ideal of a ternary semiring $S$ is a ternary subsemiring of $S$.

Lemma 3.3. Every left, right, and lateral ideal of a ternary semiring $S$ is a quasi-ideal of $S$. 
Remark 3.4. The converse of Lemma 3.3 is not true, in general, that is, a quasi-ideal may not be a left, a right, or a lateral ideal of $S$. This follows from the following example.

Example 3.5. Let $S=M_{2}\left(Z_{0}^{-}\right)$be the ternary semiring of the set of all $2 \times 2$ square matrices over $Z_{0}^{-}$, the set of all nonpositive integers. Let $Q=\left\{\left(\begin{array}{cc}a & 0 \\ 0 & 0\end{array}\right): a \in Z_{0}^{-}\right\}$. Then we can easily verify that $Q$ is a quasi-ideal of $S$, but $Q$ is not a right ideal, a lateral ideal, or a left ideal of $S$.

PROPOSITION 3.6. If $Q$ is a quasi-ideal of a ternary semiring $S$ and $T$ is a ternary subsemiring of $S$, then $Q \cap T$ is a quasi-ideal of $T$.

Lemma 3.7. The intersection of arbitrary collection of quasi-ideals of a ternary semiring $S$ is a quasi-ideal of $S$.

THEOREM 3.8. An additive subsemigroup $Q$ of a ternary semiring $S$ is a quasi-ideal of $S$ if $Q$ is the intersection of a right ideal, a lateral ideal, and a left ideal of $S$.

Proof. Let $R$ be a right ideal, $M$ be a lateral ideal, and $L$ be a left ideal of $S$ such that $Q=R \cap M \cap L$. Then, by Lemmas 3.3 and 3.7, we find that $Q$ is a quasi-ideal of $S$.

The converse of Theorem 3.8 does not hold, in general. But, in particular, we have the following result.

Theorem 3.9. An additive subsemigroup $Q$ of a ternary semiring $S$ is a minimal quasi-ideal of $S$ if and only if $Q$ is the intersection of a minimal right ideal, a minimal lateral ideal, and a minimal left ideal of $S$.

Proof. Let $R$ be a minimal right ideal, $M$ a minimal lateral ideal, and $L$ a minimal left ideal of $S$ such that $Q=R \cap M \cap L$. Then, by Theorem 3.8, it follows that $Q$ is a quasi-ideal of $S$. Now it remains to show that $Q$ is minimal. If possible, let $Q^{\prime} \subseteq Q$ be any other quasi-ideal of $S$. Then, $Q^{\prime} S S$ is a right ideal of $S$ and $Q^{\prime} S S \subseteq Q S S \subseteq R S S \subseteq R$. Since $R$ is a minimal right ideal of $S$, we have $Q^{\prime} S S=R$. Similarly, we can prove that $S Q^{\prime} S+S S Q^{\prime} S S=M$ and $S S Q^{\prime}=$ $L$. Therefore, $Q=R \cap M \cap L=Q^{\prime} S S \cap\left(S Q^{\prime} S+S S Q^{\prime} S S\right) \cap S S Q^{\prime} \subseteq Q^{\prime}$. Consequently, $Q=$ $Q^{\prime}$ and hence $Q$ is a minimal quasi-ideal of $S$.

Conversely, let $Q$ be a minimal quasi-ideal of $S$. Then, $Q S S \cap(S Q S+S S Q S S) \cap S S Q \subseteq$ Q. Let $q \in Q$. Then, $q S S$ is a right ideal, $(S q S+S S q S S)$ is a lateral ideal, and $S S q$ is a left ideal of $S$. Therefore, by Theorem 3.8, $q S S \cap(S q S+S S q S S) \cap S S q$ is a quasi-ideal of $S$, and $q S S \cap(S q S+S S q S S) \cap S S q \subseteq Q S S \cap(S Q S+S S Q S S) \cap S S Q \subseteq Q$. Since $Q$ is a minimal quasi-ideal of $S$, we have $q S S \cap(S q S+S S q S S) \cap S S q=Q$. Now it remains to show that $q S S,(S q S+S S q S S)$, and $S S q$ are, respectively, a minimal right, a minimal lateral, and a minimal left ideal of $S$. If possible, let $R$ be any right ideal of $S$ such that $R \subseteq q S S$. Then $R S S \subseteq R \subseteq q S S$. Now, $R S S \cap(S q S+S S q S S) \cap S S q \subseteq q S S \cap(S q S+S S q S S) \cap S S q=Q$. Thus, by minimality of $Q$, we find that $Q=R S S \cap(S q S+S S q S S) \cap S S q$. This implies that $Q \subseteq R S S$. Again, $q S S \subseteq Q S S \subseteq(R S S) S S \subseteq R S S$. Thus, $q S S=R S S \subseteq R$ and hence $R=q S S$. Consequently, $q S S$ is a minimal right ideal of $S$. Similarly, we can prove that $(S q S+S S q S S)$ is a minimal lateral ideal and $S S q$ is a minimal left ideal of $S$.

Proposition 3.10. Any minimal lateral ideal of a ternary semiring $S$ is a minimal ideal of $S$. 
Proof. Let $M$ be a minimal lateral ideal of $S$. We will show that $M$ is a minimal ideal of $S$. Let $m \in M$. Then, $S m S+S S m S S$ is a lateral ideal of $S$ and $S m S+S S m S S \subseteq S M S+S S M S S \subseteq$ $M$. Since $M$ is minimal, we have $M=S m S+S S m S S$. Now, $M S S=(S m S+S S m S S) S S=$ $(S m S) S S+(S S m S S) S S \subseteq S m S+S S m S S \subseteq M$ and $S S M=S S(S m S+S S m S S)=S S(S m S)+$ $S S(S S m S S) \subseteq S m S+S S m S S \subseteq M$. This implies that $M$ is both right ideal and left ideal of $S$. Consequently, $M$ is an ideal of $S$. Now it remains to show that $M$ is a minimal ideal of $S$. If possible, let $M^{\prime}$ be an ideal of $S$ such that $M^{\prime} \subseteq M$. Since $M^{\prime}$ is an ideal of $S$, it is a lateral ideal of $S$. By hypothesis, we have $M^{\prime}=M$. Consequently, $M$ is a minimal ideal of $S$.

Corollary 3.11. Any minimal quasi-ideal of a ternary semiring $S$ is contained in a minimal ideal of $S$.

Proof. Let $Q$ be a minimal quasi-ideal of $S$. Then, by Theorem 3.9, $Q=R \cap M \cap L$, where $R$ is a minimal right ideal, $M$ a minimal lateral ideal, and $L$ a minimal left ideal of $S$. Clearly, $Q \subseteq M$. From Proposition 3.10, it follows that $M$ is a minimal ideal of $S$.

Proposition 3.12. Let $x$ be an idempotent element of a ternary semiring $S$, that is, $x^{3}(=$ $x x x)=x$. If $R$ is a right ideal, $M$ a lateral ideal, and $L$ a left ideal of $S$, then $R x x, x x M x x$, and $x \times L$ are quasi-ideals of $S$.

Proof. To show $R x x, x x M x x$, and $x x L$ are quasi-ideals of $S$, it is sufficient to show that

$$
\begin{gathered}
R x x=R \cap(S x S+S S x S S) \cap S S x, \\
x x M x x=x S S \cap M \cap S S x, \\
x x L=x S S \cap(S x S+S S x S S) \cap L .
\end{gathered}
$$

For the first case, clearly we see that $R x x \subseteq R \cap S S x$. Let $a \in R \cap S S x$. Then, $a \in R$ and $a \in S S x$. Now, $a \in S S x$ implies that $a=\sum_{i=1}^{n} s_{i} t_{i} x$ for some $s_{i}, t_{i} \in S$. Therefore, $a x x=$ $\left(\sum_{i=1}^{n} s_{i} t_{i} x\right) x x=\sum_{i=1}^{n} s_{i} t_{i}(x x x)=\sum_{i=1}^{n} s_{i} t_{i} x=a$. Thus, it follows that $a \in R x x$ and hence $R x x=R \cap S S x$. Again, $a=a x x \in S x S$ and $0 \in S S x S S$. So we find that $a \in(S x S+S S x S S)$. Thus, $R \cap S S x \subseteq(S x S+S S x S S)$. Consequently, $R x x=R \cap(S x S+S S x S S) \cap S S x$.

For the second case, We see that $x x M x x \subseteq x S S \cap M \cap S S x$. Let $a \in x S S \cap M \cap S S x$. Then, $a \in x S S, a \in M$, and $a \in S S x$. Now, $a \in x S S$ and $a \in S S x$ imply that $a=\sum_{i=1}^{m} x s_{i} t_{i}=$ $\sum_{j=1}^{n} u_{j} v_{j} x$ for some $s_{i}, t_{i}, u_{j}, v_{j} \in S$. Therefore,

$$
\begin{aligned}
x x a x x & =x x\left(\sum_{i=1}^{m} x s_{i} t_{i}\right) x x=\left(\sum_{i=1}^{m}(x x x) s_{i} t_{i}\right) x x=\left(\sum_{i=1}^{m} x s_{i} t_{i}\right) x x \\
& =\left(\sum_{j=1}^{n} u_{j} v_{j} x\right) x x=\sum_{j=1}^{n} u_{j} v_{j}(x x x)=\sum_{j=1}^{n} u_{j} v_{j} x=a .
\end{aligned}
$$

Consequently, $a \in x x M x x$ and hence $x x M x x=x S S \cap M \cap S S x$.

The third case can be proved in the same way as in the first case.

We recall the definition of regular ternary semiring. 
A ternary semiring $S$ is called regular if for every $a \in S$, there exists an $x$ in $S$ such that $a x a=a$.

THeOREM 3.13. If, for every quasi-ideal $Q$ of $S, Q^{3}=Q$, then $S$ is a regular ternary semiring. Proof. If $R$ is a minimal right ideal, $M$ a minimal lateral ideal, and $L$ a minimal left ideal of $S$, then, by Theorem 3.9, it follows that $R \cap M \cap L$ is a quasi-ideal of $S$.

Now, by hypothesis,

$$
\begin{aligned}
R \cap M \cap L & =(R \cap M \cap L)^{3} \\
& =(R \cap M \cap L)(R \cap M \cap L)(R \cap M \cap L) \subseteq R M L .
\end{aligned}
$$

Again, clearly $R M L \subseteq R \cap M \cap L$. So, $R \cap M \cap L=R M L$ and hence, by [8, Theorem 3.4], $S$ is a regular ternary semiring.

Definition 3.14. A ternary subsemiring $B$ of a ternary semiring $S$ is called a bi-ideal of $S$ if $B S B S B \subseteq B$.

Lemмa 3.15. Every quasi-ideal of a ternary semiring $S$ is a bi-ideal of $S$.

Proof. Let $Q$ be a quasi-ideal of $S$. Then we see that $Q S Q S Q \subseteq Q(S S S) S \subseteq Q S S, Q S Q S Q \subseteq$ $S(S S S) Q \subseteq S S Q$, and $Q S Q S Q \subseteq S S Q S S$. Again $\{0\} \subseteq S Q S$. So, $Q S Q S Q \subseteq S Q S+S S Q S S$. Consequently, it follows that $Q S Q S Q \subseteq Q S S \cap(S Q S+S S Q S S) \cap S S Q \subseteq Q$ and hence $Q$ is a bi-ideal of $S$.

Note 3.16. The converse of Lemma 3.15 does not hold, in general, that is, a bi-ideal of a ternary semiring $S$ may not be a quasi-ideal of $S$.

Remark 3.17. Since every left, right, and lateral ideal of $S$ is a quasi-ideal of $S$, it follows that every left, right, and lateral ideal of $S$ is a bi-ideal of $S$, but the converse is not true, in general.

Proposition 3.18. If $B$ is a bi-ideal of a ternary semiring $S$ and $T$ is a ternary subsemiring of $S$, then $B \cap T$ is a bi-ideal of $T$.

LEMMA 3.19. If $B$ is a bi-ideal of a ternary semiring $S$ and $T_{1}, T_{2}$ are two ternary subsemirings of $S$, then $B T_{1} T_{2}, T_{1} B T_{2}$, and $T_{1} T_{2} B$ are bi-ideals of $S$.

Corollary 3.20. If $B_{1}, B_{2}$, and $B_{3}$ are three bi-ideals of a ternary semiring $S$, then $B_{1} B_{2} B_{3}$ is a bi-ideal of $S$.

Corollary 3.21. If $Q_{1}, Q_{2}$, and $Q_{3}$ are three quasi-ideals of a ternary semiring $S$, then $Q_{1} Q_{2} Q_{3}$ is a bi-ideal of $S$.

In general, if $B$ is a bi-ideal of a ternary semiring $S$ and $C$ is a bi-ideal of $B$, then $C$ is not a bi-ideal of $S$. But, in particular, we have the following result.

Theorem 3.22. Let $B$ be a bi-ideal of a ternary semiring $S$, and $C$ a bi-ideal of $B$ such that $C^{3}=C$. Then $C$ is a bi-ideal of $S$.

Proof. Since $B$ is a bi-ideal of $S, B S B S B \subseteq B$, and since $C$ is a bi-ideal of $B, C B C B C \subseteq C$. 
3020 On quasi-ideals and bi-ideals in ternary semirings

Therefore,

$$
\begin{aligned}
C S C S C & =(C C C) S C S(C C C) \\
& =C C(C S C S C) C C \subseteq C C(B S B S B) C C \subseteq C C B C C \\
& =C C B C(C C C) \subseteq C(C B C B C) C \subseteq C C C=C .
\end{aligned}
$$

We recall the definition of ternary division semiring.

A ternary semiring $S$ with $|S| \geq 2$ is called a ternary division semiring if for any nonzero element $a$ of $S$, there exists a nonzero element $b$ in $S$ such that $a b x=b a x=x a b=x b a=x$ for all $x \in S$.

Theorem 3.23. A ternary semiring $S$ has no nonzero proper bi-ideals if $S$ is a ternary division semiring.

Proof. Let $S$ be a ternary division semiring and $B$ be a nonzero bi-ideal of $S$. Let $a(\neq$ $0) \in B$. Then there exists $s(\neq 0) \in S$ such that $a s x=\operatorname{sax}=x a s=x s a=x$ for all $x \in S$. This implies that $S=B S S=S S B$. Now, $S=B S S=B(S S B)(S S B)=B(B S S)(S B S)(S S B) B \subseteq$ $B(B S B S B) B \subseteq B B B \subseteq B$. Consequently, $B=S$ and hence $S$ has no nonzero proper biideals.

The converse of Theorem 3.23 is not true, in general. However, in particular, we have the following result.

Theorem 3.24. A ternary semiring $S$ is a ternary division semiring if $S$ is $M C$ and has no nonzero proper bi-ideals.

Proof. Let $S$ be an MC ternary semiring and has no nonzero proper bi-ideals. Let $a(\neq$ $0) \in S$. Then, $a S x$ and $x a S$ are two bi-ideals of $S$ for any nonzero $x \in S$. Since $S$ is MC, it is ZDF. So, $a S x \neq\{0\}$ and $x a S \neq\{0\}$. By hypothesis, we have $a S x=x a S=S$ and hence for $x(\neq 0) \in S$, there exist $b, c \in S$ such that $a b x=x a c=x$. Let $y$ be any element of $S$. Then there exist $d, e \in S$ such that $a d x=x a e=y$. Thus, $a b y=a b(x a e)=(a b x) a e=x a e=y$ for all $y \in S$. Now, $(y a b) a b=y(a b a) b=y a b$. Since $S$ is MC, we find that $y a b=y$ for all $y \in S$. Similarly, we can show that $b a y=y b a=y$ for all $y \in S$. Thus, we find that $a b y=y a b=b a y=y b a=y$ for all $y \in S$, and hence $S$ is a ternary division semiring.

Proposition 3.25. Let $X, Y$, and $Z$ be three ternary subsemirings of a ternary semiring $S$ and $B=X Y Z$. Then, $B$ is a bi-ideal if at least one of $X, Y, Z$ is a right, a lateral, or a left ideal of $S$.

Proof. Let $B=X Y Z$. Suppose $X$ is a right ideal of $S$. Then we find that

$$
(X Y Z) S(X Y Z) S(X Y Z)=X(S S S)(S S S) S S Y Z \subseteq X(S S S) S Y Z \subseteq(X S S) Y Z \subseteq X Y Z .
$$

Consequently, $B=X Y Z$ is a bi-ideal of $S$.

Now suppose that $Y$ is a right ideal of $S$. Then

$$
(X Y Z) S(X Y Z) S(X Y Z) \subseteq X Y(S S S)(S S S) S S Z \subseteq X Y(S S S) S Z \subseteq X Y S S Z \subseteq X Y Z
$$

This implies that $B=X Y Z$ is a bi-ideal of $S$. 
Again, if $Z$ is a right ideal of $S$, then

$(X Y Z) S(X Y Z) S(X Y Z) \subseteq(X Y Z)(S S S)(S S S) S S \subseteq(X Y Z)(S S S) S \subseteq X Y(Z S S) \subseteq X Y Z$

Consequently, $B=X Y Z$ is a bi-ideal of $S$.

Similar proofs can be given for other cases.

Corollary 3.26. A ternary subsemiring $B$ of $S$ is a bi-ideal of $S$ if $B=R M L$, where $R$ is a right ideal, $M$ is a lateral ideal, and $L$ is a left ideal of $S$.

Proposition 3.27. Let $B$ be a ternary subsemiring of a ternary semiring $S$. If $R$ is a right ideal, $M$ is a lateral ideal, and $L$ is a left ideal of $S$ such that $R M L \subseteq B \subseteq R \cap M \cap L$, then $B$ is a bi-ideal of $S$.

Proof.

$$
B S B S B \subseteq(R \cap M \cap L) S(R \cap M \cap L) S(R \cap M \cap L) \subseteq R(S M S) L \subseteq R M L \subseteq B .
$$

The following theorem gives a characterization of a regular ternary semiring $S$ in terms of bi-ideal and quasi-ideal of $S$.

THEOREM 3.28. The following conditions in a ternary semiring $S$ are equivalent:

(i) $S$ is regular,

(ii) for every bi-ideal $B$ of $S, B S B S B=B$,

(iii) for every quasi-ideal $Q$ of $S, Q S Q S Q=Q$.

Proof. (i) $\Rightarrow$ (ii). Suppose $S$ is regular. Let $B$ be a bi-ideal of $S$. Let $b \in B$. Then there exists $x \in S$ such that $a=a x a$. This implies that $a=\operatorname{axaxa} \in B S B S B$. So we find that $B \subseteq B S B S B$. Again, since $B$ is a bi-ideal of $S, B S B S B \subseteq B$. Consequently, $B S B S B=B$.

Clearly, (ii) $\Rightarrow$ (iii), by using Lemma 3.15.

(iii) $\Rightarrow$ (i). Suppose (iii) holds. Let $R$ be a right ideal, $M$ a lateral ideal, and $L$ a left ideal of $S$. Then, $Q=R \cap M \cap L$ is a quasi-ideal of $S$, by Theorem 3.8. By hypothesis, $Q S Q S Q=Q$. Now, $R \cap M \cap L=Q=Q S Q S Q \subseteq R S M S L \subseteq R M L$. Again, clearly $R M L \subseteq R \cap M \cap L$. So, $R \cap M \cap L=R M L$, and hence, by [8, Theorem 3.4], $S$ is a regular ternary semiring.

Theorem 3.29. A ternary subsemiring $B$ of a regular ternary semiring $S$ is a bi-ideal of $S$ if and only if $B=B S B$.

Proof. If $B=B S B$, then it is easy to see that $B$ is a bi-ideal of $S$.

Conversely, suppose that $B$ is a bi-ideal of a regular ternary semiring $S$. Let $b \in B$, then there exists $x \in S$ such that $b=b x b$. This implies that $b \in B S B$ and hence $B \subseteq B S B$. Again, $B S B \subseteq B S B S B \subseteq B$. Thus we find that $B=B S B$.

Theorem 3.30. A ternary subsemiring $B$ of a regular ternary semiring $S$ is a bi-ideal of $S$ if and only if $B$ is a quasi-ideal of $S$.

Proof. Let $S$ be a regular ternary semiring. If $B$ is a quasi-ideal of $S$, then, from Lemma 3.15 , it follows that $B$ is a bi-ideal of $S$. 
Conversely, let $B$ be a bi-ideal of $S$. From [8, Theorem 3.4], we find that if $S$ is a regular ternary semiring, then $R \cap M \cap L=R M L$ for any right ideal $R$, any lateral ideal $M$, and any left ideal $L$.

Now,

$$
\begin{aligned}
B S S \bigcap(S B S & +S S B S S) \bigcap S S B \\
& =B S S(S B S+S S B S S) S S B \\
& =B(S S S) B(S S S) B+B(S S S) S B(S S S) S B \\
& \subseteq B S B S B+B S S B S S B \\
& \subseteq B+B S B \quad(\text { since } B \text { is a bi-ideal }) \\
& =B+B \quad(\text { by Theorem } 3.29) \\
& \subseteq B .
\end{aligned}
$$

Consequently, $B$ is a quasi-ideal of $S$.

In view of Lemma 3.19 and Theorem 3.30, we have the following result.

THeOREM 3.31. If $Q_{1}$ and $Q_{2}$ are two ternary subsemiring and $Q_{3}$ is a bi-ideal of a regular ternary semiring $S$, then $Q_{1} Q_{2} Q_{3}, Q_{1} Q_{3} Q_{2}$, and $Q_{3} Q_{1} Q_{2}$ are quasi-ideals of $S$.

In view of Corollary 3.21 and Theorem 3.31, we have the following result.

Corollary 3.32. For any three quasi-ideals $Q_{1}, Q_{2}, Q_{3}$ of a regular ternary semiring $S$, $Q_{1} Q_{2} Q_{3}$ is a quasi-ideal of $S$.

\section{Acknowledgments}

The author is grateful to Professor T. K. Dutta for his valuable suggestions and continuous help throughout the preparation of this note. The author is thankful to CSIR, India, for financial assistance.

\section{References}

[1] V. N. Dixit and S. Dewan, A note on quasi and bi-ideals in ternary semigroups, Int. J. Math. Math. Sci. 18 (1995), no. 3, 501-508.

[2] T. K. Dutta and S. Kar, On regular ternary semirings, Advances in Algebra, Proceedings of the ICM Satellite Conference in Algebra and Related Topics, World Scientific, New Jersey, 2003, pp. 343-355.

[3] - On ternary semifields, Discuss. Math. Gen. Algebra Appl. 24 (2004), no. 2, 185-198.

[4] On the Jacobson radical of a ternary semiring, Southeast Asian Bull. Math. 28 (2004), no. 1, 1-13.

[5] A note on the Jacobson radical of a ternary semiring I, Southeast Asian Bull. Math. 29 (2005), no. 2, 321-331.

[6] - On prime ideals and prime radical of ternary semirings, Bull. Calcutta Math. Soc. 97 (2005), no. 5, 445-454.

[7] - On semiprime ideals and irreducible ideals of ternary semirings, Bull. Calcutta Math. Soc. 97 (2005), no. 5, 467-476.

[8] A note on regular ternary semirings, personal communication. 
[9] R. A. Good and D. R. Hughes, Associated groups for a semigroup, Bull. Amer. Math. Soc. 58 (1952), 624-625.

[10] F. M. Sioson, Ideal theory in ternary semigroups, Math. Japon. 10 (1965), 63-84.

[11] O. Steinfeld, Über die Quasiideale von Halbgruppen, Publ. Math. Debrecen 4 (1956), 262-275 (German).

[12] _ Über die Quasiideale von Ringen, Acta Sci. Math. (Szeged) 17 (1956), 170-180 (German).

S. Kar: Department of Pure Mathematics, University of Calcutta, 35 Ballygunge Circular Road, Kolkata 700019, India

E-mail address: karsukhendu@yahoo.co.in 


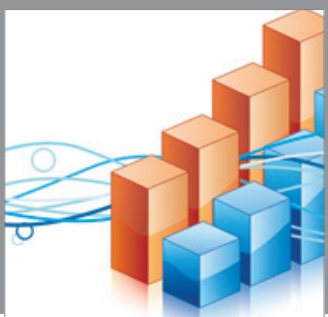

Advances in

Operations Research

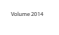

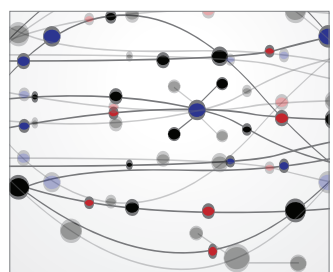

\section{The Scientific} World Journal
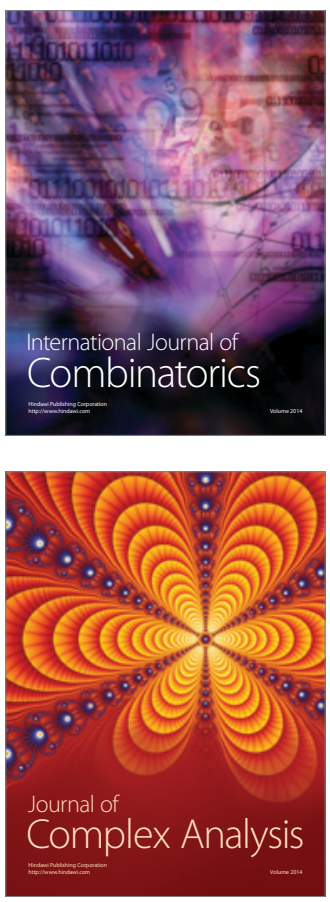

International Journal of

Mathematics and

Mathematical

Sciences
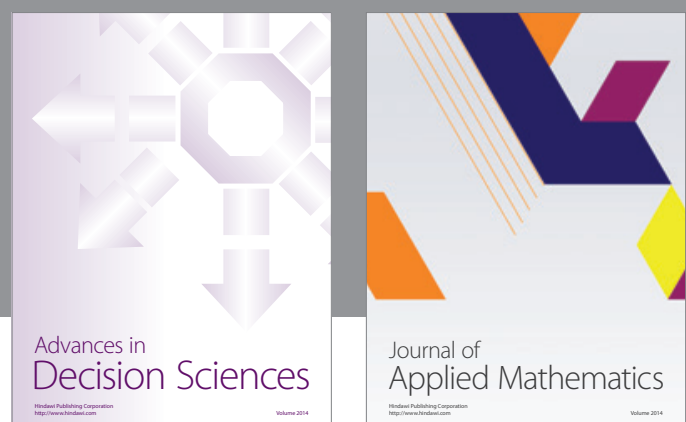

Journal of

Applied Mathematics
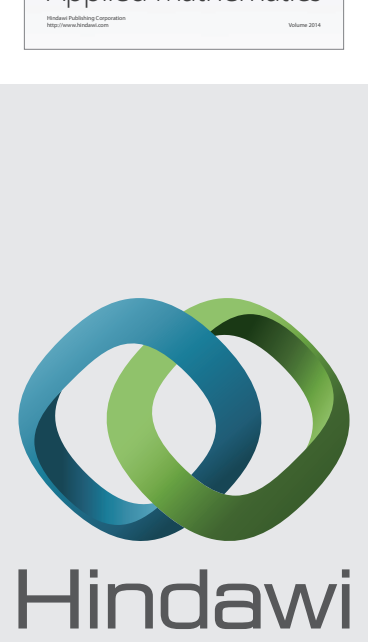

Submit your manuscripts at http://www.hindawi.com
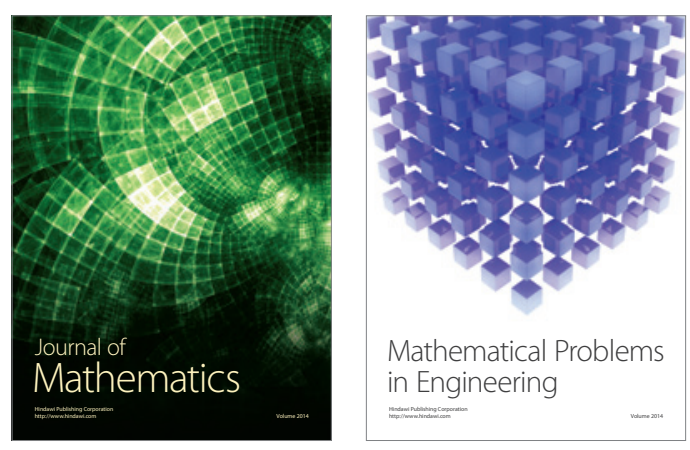

Mathematical Problems in Engineering
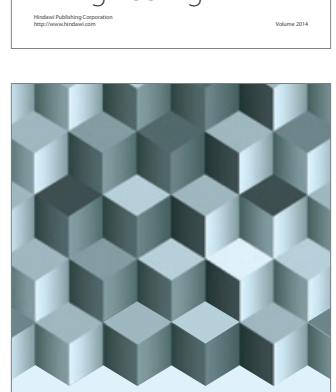

Journal of

Function Spaces
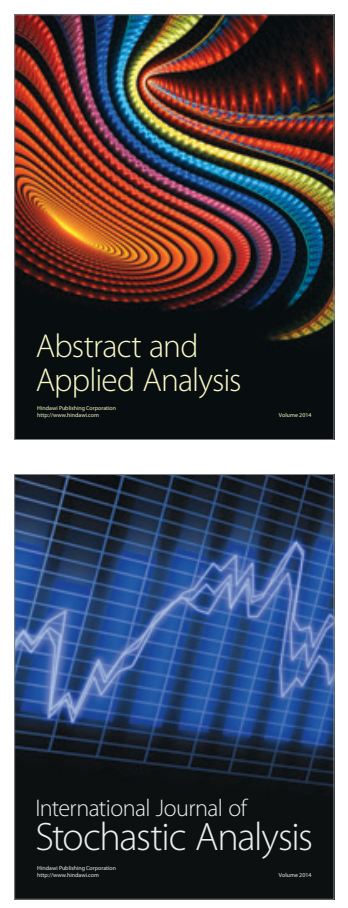

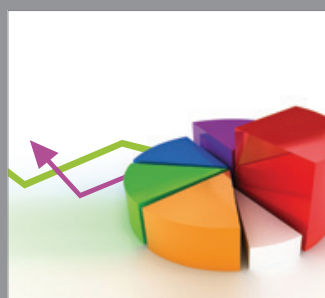

ournal of

Probability and Statistics

Promensencen
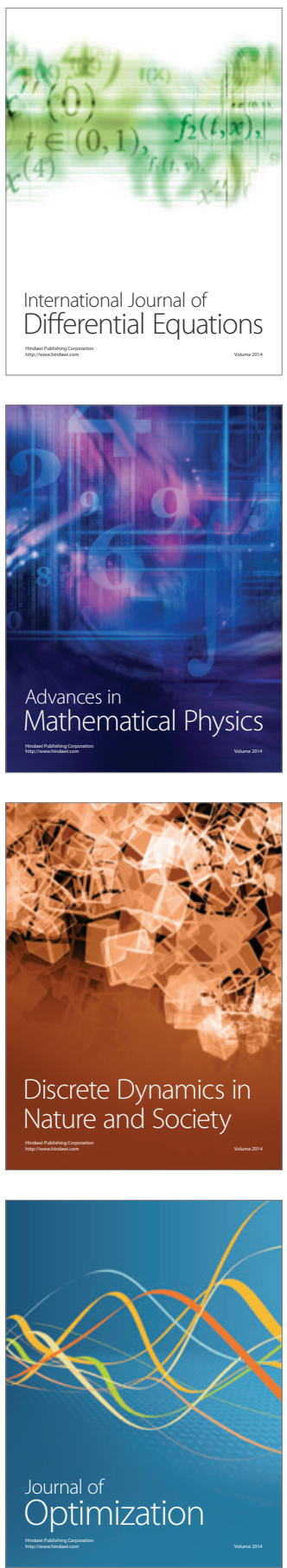